\title{
XXXI. Bars and wires of varying elasticity
}

\section{Chree B.A.}

To cite this article: C. Chree B.A. (1886) XXXI. Bars and wires of varying elasticity, Philosophical Magazine Series 5, 22:136, 259-270, DOI: 10.1080/14786448608627929

To link to this article: http://dx.doi.org/10.1080/14786448608627929

册 Published online: 29 Apr 2009.

Submit your article to this journal $2 \pi$

Џ Article views: 2

Q View related articles $\sqsubset$ 


\section{[ 259 ]}

XXXI. Bars and Wires of varying Elasticity. By C. Chree, B.A., Fellow of King's College, Cambridge*.

$\mathrm{N}$ a previous papert I considered some cases of bars and wires whose elastic properties, though constant over a cross section, varied from point to point of their lengtb. In the present paper a similar treatment is applied to certain cases where the elastic properties vary with the distance from the centre of the circular cross section, but are independent of the distance of the cross section from the ends. Before entering on this question, however, I wish to make some remarks on the trustworthiness of the method employed in my last and in the present paper.

The mathematical determination of stress as a function of strain is based upon a consideration of the molecular forces operating at points in the interior of a solid. A difference of opinion as to the nature of these forces has separated elasticians into two schools, who differ as to the number of independent elastic constants. Both schools, however, appear to agree in assuming the same relation between stress and strain to hold at the surface of a solid body as in its interior. On this assumption, generally tacitly made, are based the ordinary surface-equations whose validity seems to have been seldom questioned.

The phenomena existing at the common surface of two distinct elastic solid media have not been considered by many mathematicians. Green, however, in his celebrated papers on light, and others have supposed the same relations between stress and strain to exist in the surface-layers of each medium as in its interior, and this was assumed without comment in my previous paper.

It is, however, apparent, that there will be in, say the first medium, a surface-layer of molecules within the range of molecular action of the molecules of the second medium. Thus, when one of these molecules in the first medium is displaced, the change in the molecular force acting on it will be due partly to the action of the molecules of that medium, and partly to the action of those of the second medium. It is thus by no means obvious that the resultant change of molecular force (i. e. the stress) will depend only on the elastic constants of the first medium. We may in fact imagine two surfaces constructed, the one in the first medium and the other in the second, between which the molecules are subjected to forces which have their seat in both media; and to this, extremely limited, region the strict applicability of the

* Communicated by the Author.

+ Phil. Mag. Feb. 1886, p. 81. 
ordinarily accepted equations seems to me liable at least to criticism.

Since, however, the range of molecular action, and thus the distance between these two imaginary surfaces, must be extremely small, the elastic stresses over these surfaces must form equilibrating systems ; and these systems of stresses are, without any assumption, each expressible by the ordinary formulæ. It would thus appear that the ordinarily accepted equations between the stresses at the common surface of two media are, on the whole, as trustworthy as the three equations usually given for the stresses at the surface of a single medium.

In the case of media of finite thickness, the equations I have applied would thus appear as reliable as the ordinary equations for a single medium; but their extension to a continuously varying medium is open to objection. If, however, as in the cases worked out in my previous paper and in the present, the change be so gradual as to be inappreciable at distances of the same order as the range of molecular action, the results obtained should be, at least for practical purposes, comparatively satisfactory.

If, as in the present paper, the surfaces of separation be not plane, but cylindrical, no fresh difficulty is introduced, as the range of molecular action may be regarded as vanishing compared to the radii of curvature.

There are a great variety of structures whose elastic properties vary with the distance from a central axis, though the same at corresponding points in all cross sections. Such a condition of matters is sometimes intentionally produced; as when a bar, solid or hollow, is protected from the action of certain fluids or gases by a coating of some other material. The effect may also be due to the gradual operation of natural agents, as when girders are exposed to the action of air or water. Sometimes the process of manufacture involves such variation in the material. Thus the process of drawing increases the density and tenacity of the surface portions of a wire, which form a sort of rind to the inner and softer portions. Again, in metal casts of large cross section, it is well known that the surface-portions differ very markedly from the interior, and that in particular the strength does not by any means increase as fast as the cross section.

It thus seems desirable to find a solution for the equilibrium of a cylindrical bar exposed to longitudinal traction or pressure, or to torsion, the bar being composed of two or more materials, or of one continuously varying material, such that surfaces of equal elasticity are cylinders coaxial with the onter and inner surfaces of the bar. 
For this end the fundamental equations of my previous paper will suffice, and a reference to them will be denoted by the letter a attached to the number of the equation. The notation of that paper is also followed in the present.

Let us, then, suppose a straight bar, of length $l$ and uniform cross section, to be composed of two different materials; the elastic constants of the inner material being $m, n$, and those of the outer $m_{1}, n_{1}$. For greater generality we may suppose the bar to be hollow, its inner surface being the cylinder $r=b$; the surface of separation and the outer surface are coaxial cylinders whose radii are $a_{1}$ and $a$ respectively.

From (4a), (12a), (13a), and (14a) we see that suitable solutions are, in the case of longitudinal traction or pressure, for the inner material,

and for the outer,

$$
\begin{aligned}
& \left.\begin{array}{l}
\delta=2 \mathrm{~A}+\mathrm{B}, \\
u=\mathrm{A} r+\frac{\mathrm{A}^{\prime}}{r} \\
w=\mathrm{B} z ;
\end{array}\right\}, \cdot \cdot \cdot \cdot . \\
& \left.\begin{array}{l}
\delta_{1}=2 \mathrm{~A}_{1}+\mathrm{B}, \\
u_{1}=\mathrm{A}_{1} r+\frac{\mathrm{A}_{1}^{\prime}}{r}, \\
w_{1}=\mathrm{B} z ;
\end{array}\right\} \cdot \cdot \cdot \cdot \cdot
\end{aligned}
$$

where $\mathrm{B}, \mathrm{A}, \mathrm{A}^{\prime}, \mathrm{A}_{1}, \mathrm{~A}_{1}{ }^{\prime}$ are constants to be determined from the surface-conditions. We shall suppose the bar fixed at the end $z=0$ so that $w$ and $w_{1}$ both must vanish with $z$. It is then obvious that if the materials are to stick together throughont, $\mathrm{B}$ must be the same in the expressions for $w$ and for $w_{1}$. At each of the three surfaces $r=b, r=a_{1}, r=a$ we have to satisfy the equations (10a), putting $\lambda=1$ and $\mu=\nu=0$. Now the only stresses corresponding to the solution (1) are

$$
\begin{aligned}
& \mathrm{P}=(m-n) \delta+2 n \frac{d u}{d r}=(m-n) \mathrm{B}+2 m \mathrm{~A}-\frac{2 n}{r^{2}} \mathrm{~A}^{\prime}, . \\
& \mathrm{R}=(m-n) \delta+2 n \frac{d w}{d z}=(m+n) \mathrm{B}+2(m-n) \mathrm{A} ; .
\end{aligned}
$$

and to the solution (2) in like manner,

$$
\begin{aligned}
& \mathrm{P}_{1}=\left(m_{1}-n_{1}\right) \mathrm{B}+2 m_{1} \mathrm{~A}_{1}-\frac{2 n_{1}}{r^{2}} \mathrm{~A}_{1}^{\prime}, . . . \\
& \mathrm{R}_{1}=\left(m_{1}+n_{1}\right) \mathrm{B}+2\left(m_{1}-n_{1}\right) \mathrm{A}_{1} . . .
\end{aligned}
$$

Thus the equations (10a) give, neglecting any surface normal forces such as atmospheric pressure, which can, how- 
ever, if desired, be easily considered separately, when $r=b, \mathrm{P}=0$, i. $e$.

$$
(m-n) \mathrm{B}+2 m \mathrm{~A}-\frac{2 n}{b^{2}} \mathrm{~A}^{\prime}=0, . .
$$

when $r=a, \mathrm{P}_{1}=0$, i. $e$.

$$
\left(m_{1}-n_{1}\right) \mathrm{B}+2 m_{1} \mathrm{~A}_{1}-\frac{2 n_{1}}{a^{2}} \mathrm{~A}_{1}^{\prime}=0 ;
$$

and when $r=a_{1}$,

$$
(m-n) \mathrm{B}+2 m \mathrm{~A}-\frac{2 n}{a_{1}^{2}} \mathrm{~A}^{\prime}=\left(m_{1}-n_{1}\right) \mathrm{B}+2 m_{1} \mathrm{~A}_{1}-\frac{2 n_{1}}{a_{1}^{2}} \mathrm{~A}_{1}^{\prime} \text {. }
$$

At this last surface also the radial displacements in the two media must be equal ; therefore

From (10),

$$
\mathrm{A} a_{1}+\frac{\mathrm{A}^{\prime}}{a_{1}}=\mathrm{A}_{1} a_{1}+\frac{\mathrm{A}_{1}^{\prime}}{a_{1}} . \quad . \quad . \quad .
$$

while from (7), (8), and (9),

$$
\mathrm{A}_{1}^{\prime}-\mathrm{A}^{\prime}=a_{1}^{2}\left(\mathbf{A}-\mathrm{A}_{1}\right)
$$

$$
n_{1} b^{2}\left(a^{2}-a_{1}{ }^{2}\right) \mathrm{A}_{1}{ }^{\prime}+n a^{2}\left(a_{1}{ }^{2}-b^{2}\right) \mathrm{A}^{\prime}=0 .
$$

Thus, if for shortness,

we get

$$
n_{1} b^{2}\left(a^{2}-a_{1}^{2}\right)+n a^{2}\left(a_{1}^{2}-b^{2}\right)=\frac{1}{\Delta_{1}}, \quad .
$$

$$
\left.\begin{array}{l}
\mathrm{A}^{\prime}=-n_{1} a_{1}^{2} b^{2}\left(a^{2}-a_{1}^{2}\right) \Delta_{1}\left(\mathrm{~A}-\mathrm{A}_{1}\right) \\
\mathrm{A}_{1}^{\prime}=n a_{1}^{2} a^{2}\left(a_{1}^{2}-b^{2}\right) \Delta_{1}\left(\mathrm{~A}-\mathrm{A}_{1}\right) .
\end{array}\right\} .
$$

If, again, for shortness,

$$
\frac{1}{\Delta_{1}}+\frac{n n_{1}}{m m_{1}} a_{1}^{2}\left\{m_{1}\left(a^{2}-a_{1}^{2}\right)+m\left(a_{1}^{2}-b^{2}\right)\right\}=\frac{1}{\Delta_{2}}, .
$$

we get by substituting the values (12) in (7) and (8), dividing these equations by $2 m$ and $2 m_{1}$ respectively, and subtracting,

where

$$
\mathrm{A}-\mathrm{A}_{1}=\mathrm{B}\left(\sigma_{1}-\sigma\right) \frac{\Delta_{2}}{\Delta_{1}}, \quad . \quad . \quad .
$$

$$
\sigma=\frac{m-n}{2 m}, \quad \sigma_{1}=\frac{m_{1}-n_{1}}{2 m_{1}} .
$$

Thus from (7) and (8), finally, we obtain

$$
\left.\begin{array}{l}
\mathrm{A}=-\mathrm{B}\left\{\sigma+\left(\sigma_{1}-\sigma\right) \frac{n n_{1}}{m} a_{1}^{2}\left(a^{2}-a_{1}^{2}\right) \Delta_{2}\right\}, \\
\mathrm{A}_{1}=-\mathrm{B}\left\{\sigma_{1}-\left(\sigma_{1}-\sigma\right) \frac{n n_{1}}{m_{1}} a_{1}^{2}\left(a_{1}^{2}-b^{2}\right) \Delta_{2}\right\}
\end{array}\right\} .
$$


Also from (12) and (14),

$$
\left.\begin{array}{l}
\mathrm{A}^{\prime}=-\mathrm{B}\left(\sigma_{1}-\sigma\right) n_{1} a_{1}^{2} b^{2}\left(a^{2}-a_{1}^{2}\right) \Delta_{2}, \\
\mathrm{~A}_{1}^{\prime}=\mathrm{B}\left(\sigma_{1}-\sigma\right) n a_{1}^{2} a^{2}\left(a_{1}^{2}-b^{2}\right) \Delta_{2} .
\end{array}\right\} .
$$

If $\mathrm{F}$ denote the total amount of traction, or negative pressure, applied over the end $z=l$ of the bar, we can determine B from the condition

$$
\mathrm{F}=\pi\left\{\left(a_{1}^{2}-b^{2}\right) \mathrm{R}+\left(a^{2}-a_{1}^{2}\right) \mathrm{R}_{1}\right\} .
$$

Referring to (4) and (6), using (15), and denoting $\frac{n}{m}(3 m-n)$ by $\mathrm{M}$, and $\frac{n_{1}}{m_{1}}\left(3 m_{1}-n_{1}\right)$ by $\mathrm{M}_{1}$, we find very simply

$$
\begin{aligned}
\mathrm{F}=\pi \mathrm{B}[ & \mathrm{M}\left(a_{1}^{2}-b^{2}\right)+\mathrm{M}_{1}\left(a^{2}-a_{1}{ }^{2}\right) \\
& \left.+4\left(\sigma_{1}-\sigma\right)^{2} n n_{1} a_{1}{ }^{2}\left(a^{2}-a_{1}{ }^{2}\right)\left(a_{1}{ }^{2}-b^{2}\right) \Delta_{2}\right] ;
\end{aligned}
$$

which determines $B$ in terms of given quantities.

It should be noticed that, strictly, F should be distributed so that there should be at every point of the terminal section in the one material the traction $R$, and in the other the traction $R_{1}$. In any practical case this is not likely actually to occur; but if the traction be applied symmetrically so that its resultant acts along the axis of the beam, and if the radius of the cross section be small compared to the length of the beam, the above solution may be regarded as sufficiently correct, except perhaps for points close to the terminal section.

From (15), (16), and (17) all the constants of the solutions (1) and (2) are expressed directly in terms of $F$ and other given quantities; thus these solutions are complete.

If, as in the case of ordinary wire, the cylinder be solid to the centre, we have $b=0$, and so

and

$$
\frac{1}{\Delta_{1}}=n a^{2} a_{1}^{2}
$$

$$
\frac{1}{\Delta_{2}}=n a_{1}^{2}\left[a^{2}+\frac{n_{1}}{m m_{1}}\left\{m_{1}\left(a^{2}-a_{1}^{2}\right)+m a_{1}^{2}\right\}\right] \text {. }
$$

Thus the expressions (15) are comparatively short; while from (16) $\mathrm{A}^{\prime}=0$, and $\mathrm{A}_{1}^{\prime}$ is a simple expression.

In the event of $\sigma_{1}$ and $\sigma$ being equal a great simplification occurs. From (16) we see that $\mathrm{A}^{\prime}$ and $\mathrm{A}_{1}{ }^{I}$ identically vanish, while from (15),

$$
\mathrm{A}=-\mathrm{B} \sigma, \quad \mathrm{A}_{1}=-\mathrm{B} \sigma_{1}=\mathrm{A} .
$$

Also from (17),

$$
\mathrm{F}=\pi \mathrm{B}\left[\mathrm{M}\left(a_{1}^{2}-b^{2}\right)+\mathrm{M}_{1}\left(a^{2}-a_{1}^{2}\right)\right] . .
$$


Thus the solutions (1) and (2) coincide, both reducing to

$$
\left.\begin{array}{l}
u=-\sigma \mathrm{B} r \\
w=\mathrm{B} z
\end{array}\right\} . \cdot \cdot \cdot \cdot \text {. }
$$

where $\mathbf{B}$ is simply determined by (18).

According to many foreign elasticians, following Poisson, $m=2 n$ for any elastic solid ; if this were correct, then necessarily $\sigma=\sigma_{1}$, and the simple solution (19) would be true for any combination of two elastic materials forming a cylinder, whether hollow or not. This theory, however, seems to be contradicted by experiment; still if, as in the case of wire, both materials are of the same metal but have been exposed to different treatment, it seems by no means unlikely that the variation of the elastic constants $m$ and $n$ will follow the same law, in which case $\sigma=\sigma_{1}$ obviously. In actual wire, of course, there is no strict surface of demarcation answering to the cylinder $r=a_{1}$ of the above problem; but in many cases the transition is very rapid, and the absolute amount of change considerable, and in such cases the previous solutions should give results comparing favourably with those obtained by neglecting the change altogether, as is usual.

The same method will apply to any number of materials in contact, the surfaces of separation being coaxial cylinders. Thus, suppose there to be $i+1$ materials whose elastic constants, proceeding outwards, are in order $(m, n),\left(m_{1}, n_{1}\right), \ldots$ $\left(m_{i}, n_{i}\right)$. Let the surfaces of separation be in order $r=a_{1} \ldots$ $r=a_{i}$, the outmost surface of all being $r=a$, and the inmost, if the cylinder be hollow, $r=b$. Employing also suffixes to distinguish the constants for the several materials, we may take as the solution for the $(s+1)$ th material, following (1),

$$
\left.\begin{array}{rl}
\delta_{s} & =2 \mathrm{~A}_{s}+\mathrm{B}, \\
u_{s} & =\mathrm{A}_{8} r+\frac{\mathrm{A}_{s}^{\prime}}{r}, \\
w_{s} & =\mathrm{B} z ;
\end{array}\right\} . . . . .
$$

where, as previously, the $B$ is the same for all the media. At the common surface $r=a_{s}$ of this and the $s$ th medium we have, precisely as in (9) and (10),

$$
\begin{array}{r}
\left(m_{s-1}-n_{s-1}\right) \mathrm{B}+2 m_{s-1} \mathrm{~A}_{s-1}-\frac{2 n_{s-1}}{a_{s}^{2}} \mathrm{~A}_{s-1}^{\prime}=\left(m_{s}-n_{s}\right) \mathrm{B} \\
+2 m_{s} \mathrm{~A}_{s}-\frac{2 n_{s}}{a_{s}^{2}} \mathrm{~A}_{s}^{\prime}, .
\end{array}
$$

and

$$
\mathrm{A}_{s-1} a_{s}+\frac{\mathrm{A}_{s-1}^{\prime}}{a_{s}}=\mathrm{A}_{s} a_{s}+\frac{\mathrm{A}_{s}^{\prime}}{a_{s}} \text {. . . . . }
$$




$$
\text { of varying Elasticity. }
$$

At each of the $i$ surfaces of separation there are two equations of the types (21) and (22). At the outmost surface $r=a$, neglecting atmospheric pressure as before, we have

$$
\left(m_{i}-n_{i}\right) \mathrm{B}+2 m_{i} \mathrm{~A}_{i}-\frac{2 n_{i}}{a^{2}} \mathrm{~A}_{i}^{\prime}=0 ; \quad . \quad .
$$

and at the inmost surface $r=b$ a similar equation, writing $b$ for $a$ and dropping the suffix. If the cylinder be not hollow this last equation does not exist, but in its place we obviously have $\mathrm{A}^{\prime}=0$. Thus there are $2 i+2$ or $2 i+1$ equations according as the cylinder is hollow or not, to determine the constants of types $A$ and $A^{\prime}$ in terms of $B$. Of these constants each medium possesses two, except when the cylinder is solid, when the central medium has only one constant, viz. A. Thus, whether the cylinder be hollow or not the equations are the same in number as the constants, which thus may all be directly expressed in terms of $B$. The values of the constants can be at once written down under the form of determinants. To determine $\mathrm{B}$, we have

$$
\mathrm{F}=\pi\left[\left(a_{1}{ }^{2}-b^{2}\right) \mathrm{R}+\left(a_{2}{ }^{2}-a_{1}{ }^{2}\right) \mathrm{R}_{1}+\ldots\left(a^{2}-a_{i}{ }^{2}\right) \mathrm{R}_{i}\right] .
$$

For the special case $\frac{m_{s}}{n_{s}}=$ constant for all values of $s$, of which we have previously seen the importance, a very simple solution holds. In fact it is obvious from (21), (22), and (23) that all our conditions are then satisfied by

$$
\left.\begin{array}{l}
\mathrm{A}_{s}^{\prime}=\mathrm{A}_{s-1}^{\prime}=\ldots=0, \\
\mathrm{~A}_{s}=\mathrm{A}_{s-1}=\ldots=-\frac{m-n}{2 m} \mathrm{~B}=-\sigma \mathrm{B}
\end{array}\right\} .
$$

in our previous notation, $\sigma$ being, in accordance with this hypothesis, the same for all the media. In this case the equation for B is simply

$$
\mathrm{F}=\pi \mathrm{B}\left[\left(a_{1}^{2}-b^{2}\right) \mathrm{M}+\left(a_{2}^{2}-a_{1}^{2}\right) \mathrm{M}_{1}+\ldots+\left(a^{2}-a_{i}^{2}\right) \mathrm{M}_{i}\right], \ldots
$$

where $M, M_{1}$, \&c. denote Young's moduli for the several media. Also the solution (19) applies to all the media.

The equations (21) and (22) have been established independently of the absolute thickness of the layers : thus, under certain limitations to be presently considered, they may be supposed to hold when the thickness is indefinitely reduced, and thus in the limit to apply to a continuously varying medium. Thus, dropping the suffixes and writing $r$ for $a_{s+1}$, we get in place of the constants of types $A$ and $A^{\prime}$ certain functions of Phil. Mag. S. 5. Vol. 22. No. 136. Sept 1886. 
$r$ determined by the equations

$$
\begin{aligned}
& 2 \frac{d}{d r}(m \mathrm{~A})+\mathrm{B} \frac{d}{d r}(m-n)-\frac{2}{r^{2}} \frac{d}{d r}\left(n \mathrm{~A}^{\prime}\right)=0, \\
& r^{2} \frac{d \mathrm{~A}}{d r}+\frac{d \mathrm{~A}^{\prime}}{d r}=0 . \quad \text {. . . . . . . . . . . . }
\end{aligned}
$$

Being given the law of variation of $m$ and $n$, we can from these equations get a differential equation in $A$ or in $A^{\prime}$ as is desired. To determine the constants of the solution, we have

$$
2 m \mathrm{~A}+(m-n) \mathbf{B}-2 n \frac{\mathbf{A}^{\prime}}{r^{2}}=0 ;
$$

when $r=a$, and also when $r=b$ if the cylinder be hollow; if it be solid, instead of the latter equation we have $A^{\prime}=0$ when $r=0$. In obtaining these equations we have, it seems to me, tacitly assumed that the force at any point is the same as if all the neighbouring material, at least on one side of a plane through the point, within the distance at which molecular forces are sensible, were the same as at the point considered. Thus, if the variation in the material were very rapid, the validity of deductions from these equations might be questioned.

As an example of the use of (26) and (27), let us consider the case of a solid cylinder of which the material has elastic constants given by

$$
\left.\begin{array}{l}
m=m_{0}(1+p r) \\
n=n_{0}(1+q r) ;
\end{array}\right\}
$$

where $m_{0}, n_{0}, p, q$ are absolute constants; while $p a: 1$ and $q a: 1$ are so small that terms containing their squares or products may be neglected. The form of (26) and (27), then, suggests

$$
\mathrm{A}=\mathrm{A}_{0}(1+c r), \cdot \cdot \cdot . \quad .
$$

where $\mathrm{A}_{0}$ and $c$ are constants, the latter being of the same order of quantities as $p$ and $q$. Then from (27) we get

$$
\mathrm{A}^{\prime}=-\mathrm{A}_{0} \frac{c r^{3}}{3}, \quad \cdot \quad \cdot \quad \cdot \quad \cdot \quad \cdot
$$

no constant being required as $A^{\prime}$ vanishes when $r=0$. Substituting (29) and (30) in (26) and retaining only the principal terms, we get

$$
\mathrm{A}_{0} c=-\frac{\mathrm{B}\left(m_{0} p-n_{0} q\right)+2 m_{0} p \mathrm{~A}_{0}}{2\left(m_{0}+n_{0}\right)} . .
$$

The surface-condition $\mathrm{P}=0$ when $r=a$ gives, when the above 
values of $m, n, \mathrm{~A}$, and $\mathrm{A}^{\prime}$ are substituted in (3),

$$
\begin{array}{r}
2 \mathrm{~A}_{0}\left[m_{5}(1+p a)+c a\left(m_{0}+\frac{1}{3} n_{0}\right)\right]=-\mathrm{B}\left[m_{0}-n_{0}\right. \\
\left.+\left(m_{0} p-n_{0} q\right) a\right] . . .
\end{array}
$$

The first approximation gives

$$
\mathrm{A}_{0}=-\sigma_{0} \mathrm{~B}
$$

Writing this in (31), we get

$$
\mathrm{A}_{0} c=-\frac{\mathrm{B} n_{0}(p-q)}{2\left(m_{0}+n_{0}\right)} . \text {. . . . . }
$$

Substituting this value of $c$ in (32), we get, after reduction, for a second approximation,

$$
\mathrm{A}_{0}=-\mathrm{B}\left[\sigma_{0}+\frac{n_{0}^{2}(p-q) a}{3 m_{0}\left(m_{0}+n_{0}\right)}\right] . \quad . \quad .
$$

Introducing these values of $A_{0}, c, A^{\prime}$ inj (1), we get, after reduction,

$$
u=-\sigma_{0} \mathrm{~B} r\left[1+\frac{2}{3} \frac{n_{0}(p-q)}{m_{0}{ }^{2}-n_{0}{ }^{2}}\left(n_{0} a+m_{0} r\right)\right] ; .
$$

while throughout,

$$
w=\mathrm{B} z \text {. }
$$

It should be noticed that correct values of $P$ and $R$ are to be obtained only from (3) and (4), substituting therein the above values of $\mathrm{A}, \mathrm{A}^{\prime}, m$, and $n$.

If, as previously, $F$ denote the total traction over the terminal section, we get

$$
\mathrm{F}=\int_{0}^{a} 2 \pi r \mathrm{R} d r
$$

where $\mathbf{R}$ has the value (4), when $m, n$, and $\mathbf{A}$ are regarded as variables given by (28), (29), (33), and (34).

This equation easily leads to

$$
\mathrm{F}=\pi a^{2} \mathrm{M}_{0} \mathrm{~B}\left[1+\frac{2}{3} a q+\frac{2}{3} a(p-q) \frac{n_{0}}{3 m_{0}-n_{0}}\right],
$$

where $M_{0}$ is the value of Young's modulus for the material at the axis.

When $\frac{m}{n}$ is constant, $p=q$; and the above value of $\mathrm{B}$ obviously agrees with that derived from (25), noticing that then $\mathrm{M}=\mathrm{M}_{0}(1+q r)$.

The torsion of a cylinder, hollow or solid, formed of different materials or of one continuously varying material, as in the cases just considered, presents no difficulty. Regarding 
first a succession of materials in contact, we see from (29a) and (30a) that, supposing the end $z=0$ fixed, a possible solution for any medium is

$$
v=\mathrm{E} r z \text {. }
$$

Further, this solution, regarding $\mathrm{E}$ as an absolute constant, will apply to all the media. For from (9a) we see that the only stress existing will be $\mathrm{S}$, and at all the surfaces of separation, as well as at the bounding surfaces of the cylinder, $\mu=\nu=0$; thus all the surface-equations (10a) are identically satisfied. Also the value of $v$ is the same for any two adjacent media at their common surface. If $G$ be the couple of torsion applied at the end of the cylinder, and if $a_{1} \ldots a_{i}$, $n, n_{1} \ldots n_{i}$ bave their previous meaning, we get, to determine $\mathrm{E}$,

i. $e$.

$$
\mathrm{G}=2 \pi \mathrm{E}\left[\int_{b}^{a_{1}} n r^{3} d r+\int_{a_{1}}^{a_{2}} n_{1} r^{3} d r+\ldots+\int_{a_{i}}^{a} n r^{3} d r\right]
$$

$$
\mathrm{G}=\frac{\pi \mathrm{E}}{2}\left[n\left(a_{1}^{4}-b^{4}\right)+n_{1}\left(a_{2}^{4}-a_{1}^{4}\right)+\ldots+n_{i}\left(a^{4}-a^{4}\right)\right] .
$$

The values of $a$ and $b$ and the number of the media may be any whatever.

With limitations similar to the case of longitudinal traction this solution may be supposed to apply to a coninuously varying medium, and the value of $\mathrm{E}$ will then be given by

$$
\mathrm{G}=2 \pi \mathrm{E} \int_{b}^{a} n r^{3} d r . \text {. . . . . }
$$

This last expression obviously includes (38), treating $n$ as a discontinuous function.

The chief use of the preceding investigations would probably be in assigning the limit to the traction, pressure, or torsion which could be applied with safety to a structure of the kind considered. Unfortunately there seems no general agreement among practical men as to how the limits of safety may be fixed for a material when exposed to any system of force, except perhaps longitudinal traction. One theory that seems to meet with considerable approval is that, whatever the system of forces may be, the structure is safe so long as the greatest positive strain does not exceed a certain limit, to be determined experimentally for each separate material, presumably by uniform traction. As to the correctness of this limit for the case of uniform traction no doubt need be entertained. It does not follow that rupture will ensue as soon as this limit is passed; but the nature of the material itself will be altered, and rupture will follow sooner or later. 
In the case of traction the only positive strain is $\frac{d w}{d z}$ or $\mathrm{B}$, which is the same for all the media of which the har may consist. If, then, the traction $\mathrm{F}$ be increased till $\mathrm{B}$ exceed the limit of safety of any one material, that material will finally give way. Theoretically, of course, this material might give way uniformly all round, with the result that the traction $\mathrm{F}$ would then have to be supported by the remaining materials. This would lead to increased strain in all these; but the structure as a whole would still be safe if this new strain were less than the limit of safety for each of the materials left. If the increased strain exceeded this limit then a second rupture would occur, and so on. In practice, owing to some want of symmetry in the distribution of the traction, or to slight inequality in the material, the first yielding material would probably crack and give way only in the neighbourhood of one point. This would alter the distribution of the traction, and might bring it to bear most largely on the strongest materials. If, however, the result were that the line of action of the resultant of the tractional forces got displaced to a finite distance from the axis of the cylinder, the strain would be considerably lessened at some points and considerably increased at others. It is obvious that such local increase of strain would be extremely dangerous. Thus the traction to be applied with safety to a composite bar of this kind should be calculated on the basis of the resultant strain not exceeding the limit of safety of the material for which the limit is least.

In the case of longitudinal pressure $B$ is negative, and for a bar of one isotropic material the other two principal strains (viz. $\frac{d u}{d r}$ and $\frac{u}{r}$ ) are each equal to $-\sigma \mathrm{B}$. In accordance with the theory recently referred to, $-\sigma B$ should not exceed the limit of safety as determined for the material by longitndinal traction. Others hold that the compression (i.e. B taken numerically) should not exceed a certain independent limit obtained from pressure experiments. The results we have obtained will enable the greatest pressure to be calculated which can be applied to a composite bar, without passing the limit of safety, for any one of the materials, determined in accordance with either of the above theories.

In the case of torsion the only existent strain is the shear $\frac{d v}{d z}=\mathrm{E} r$, which is shown in any treatise on elastic solids to be equivalent to an equal extension and compression in directions making each an angle of $45^{\circ}$ with the direction of shear, the 
numerical measure of either being $\frac{1}{2} \mathrm{E} r$. Thus, according to the theory first mentioned, in the case of torsion of a composite circular bar, if the couple of torsion be such that $\frac{1}{2} \mathrm{E} r$ exceed at any distance from the axis the limit of safety, as determined by tractional experiments, for the material at that distance rupture will ensue. The rupture would at first be limited to the single material; and if it proceeded right round would merely produce an increased strain in the remaining media, which might or might not, according to circumstances, produce further rupture. In practice, rupture would in all probability be at first limited to a small region, and the bar would undoubtedly tend to become warped. Direct experiments on the rupture of isotropic bars by torsion may disprove the above theory, but it is fairly obvious that the true law must depend on the state of strain and stress in the material. Thus the preceding solution will in any case supply the data that may be necessary in determining the limit of safety of a composite bar under torsion.

XXXII. Further Remarks on Mr. Aitken's Theory of Dew. By Charles Tomlinson,"; F.R.S.*

I HAD no idea that the innocent title of my paper, "Remarks on a New Theory of Dew," had a guilty meaning; but according to Mr. Aitken I " was raising a false contention," and so attempting to place the author "in opposition to recognized authorities"; that the results of his investigation "are in no sense entitled to be called new ;" and he repeatedly states that his investigation was not promulgated in opposition to the theory of Dr. Wells, but " in extension of the work, the foundations of which were laid by Dr. Wells." Again, he says the new theory "is not in opposition, nor are the results contrary to the teaching of Dr. Wells." Once more, the author " never made any attempt to set aside Wells's theory."

And yet it is curious to notice that 'Chambers's Journal' for May 29th last contains an article headed " $A$ New Theory of Dew," in which the writer, after giving an accurate outline of Wells's theory, goes on to say that Mr. Aitken " has brought forward many observations, and the results of numerous experiments, which appear to prove that Dr. Wells' theory of dew is not, after all, correct." We are further informed that

* Communicated by the Author. 